\title{
Tuning of Multivariable Decentralized Controllers Through the Ultimate-Point Method
}

\author{
Lucíola Campestrini, Luiz Carlos Stevanatto Filho, and Alexandre Sanfelice Bazanella
}

\begin{abstract}
A successful method for tuning single-loop PID controllers is the ultimate-point method. This method is based on the identification of the ultimate point of the process' frequency response followed by its shifting, through appropriate choices of the controller's parameters, to a specified location of the complex plane. This paper presents an extension of the ultimate-point method to the tuning of decentralized controllers for multivariable processes.
\end{abstract}

Index Terms-Characteristic loci, multivariable decentralized control, PID control, relay-feedback experiment, stability margins, ultimate-point method, Ziegler-Nichols formulas.

\section{INTRODUCTION}

D ESPITE SEVERAL decades of industrial PID control practice, a large proportion of the control loops still presents poor performance [1]. For many control loops, a good tuning method is one that would require little information about the process dynamics, so that this information can be obtained by carrying out an experiment of limited complexity, followed by the application of simple tuning rules. One of the most successful methods is the one based on the knowledge of the ultimate point of the process' frequency response, i.e., the point at which its phase reaches $-180^{\circ}$. The characteristics of the frequency response at this point are usually called the ultimate quantities of the process: the ultimate period and gain. In single-input-single-output (SISO) control, well-known formulas, such as Ziegler-Nichols and Tyreus-Luyben, use these ultimate values in order to tune PI and PID controllers [1]-[3]. Different names have been given to this method; in this paper, we refer to it as the ultimate-point method.

In multivariable multiple-input-multiple-output (MIMO) processes, it is common practice to use the SISO procedure and tuning formulas, followed by a "detuning," which consists in reducing the controller gains by some ad hoc factor [4]. This gain reduction tends to enhance the SISO stability margins, so that the coupling with other loops will-hopefully-not destabilize the loop. Better tuning can be obtained by using sequential controller tuning [2], [5], but this procedure may require a large number of experiments. Other techniques have

\footnotetext{
Manuscript received November 26, 2007; revised May 20, 2008. Manuscript received in final form August 22, 2008. First published April 21, 2009; current version published October 23, 2009. Recommended by Associate Editor S.-L. Jámsá-Jounela.

L. Campestrini and A. S. Bazanella are with the Electrical Engineering Department, Universidade Federal do Rio Grande do Sul, 90035-190, Porto Alegre, RS, Brazil (e-mail: luciola@ece.ufrgs.br; bazanela@ece.ufrgs.br; bazanela@ inma.ucl.ac.be).

L. C. Stevanatto Filho is with the Mechanical Engineering Department, Universidade de Caxias do Sul, 95070-560, Caxias do Sul, RS, Brazil (e-mail: luiz.carlos@ucs.br).

Digital Object Identifier 10.1109/TCST.2008.2006495
}

been applied as well, mostly based on the SISO method and experiments [4], [6]. Other methods deal with the identification of more than one point of the frequency response followed by the controller tuning [7], [8], but these usually apply to cross-coupled (i.e., not decentralized) controllers. In addition, there are tuning methods that yield more efficient controllers but are much more demanding in terms of the data that must be collected from the process' operation [9].

More recently, extensions of the ultimate-point method for decentralized control of multivariable processes have been proposed [10]-[12]. In these papers, the multivariable nature of the problem is explicitly acknowledged: The MIMO ultimate quantities are obtained by means of multivariable experiments. However, this information is used for tuning the controllers with the SISO formulas, a procedure that does not seem substantiated by formal multivariable analysis. Moreover, MIMO processes have infinitely many ultimate points, and the resulting closed-loop performance is strongly related to the particular ultimate point used. Hence, a better understanding of the MIMO control problem and how to use the ultimate quantities to design MIMO controllers is in order [13], [14].

In this paper, we provide a consistent criterion for determining the settings of decentralized PID controllers based on the ultimate quantities identified. The tuning is made based on a multivariable frequency-response criterion: shifting the ultimate point to an assigned location in the complex plane. In so doing, like in the SISO case, adequate stability margins can be obtained, provided that the frequency response of the process satisfies certain properties. The closed-loop performance obtained with this method presents little sensitivity to the particular ultimate point used for the design.

We consider MIMO square processes described by a transfer matrix

$$
Y(s)=G(s) U(s)
$$

where $Y(s)$ and $U(s)$ are vectors with $m$ components, representing the Laplace transforms of the process output and input, respectively, and $G(s)$ is the process' transfer matrix. The processes are assumed to be bounded-input-bounded-output (BIBO) stable.

We aim at designing decentralized controllers

$$
\begin{aligned}
U(s) & =C(s) E(s) \\
& =\left[\begin{array}{ccccc}
p_{1}(s) & 0 & 0 & \cdots & 0 \\
0 & p_{2}(s) & 0 & \cdots & 0 \\
\vdots & \vdots & & \ddots & \\
0 & 0 & 0 & \cdots & p_{m}(s)
\end{array}\right]\left[\begin{array}{c}
e_{1}(s) \\
e_{2}(s) \\
\vdots \\
e_{m}(s)
\end{array}\right]
\end{aligned}
$$

where $E(s)=R(s)-Y(s), R(s)$ is the Laplace transform of the reference signal, and $p_{i}(s), i=1, \ldots, m$, 


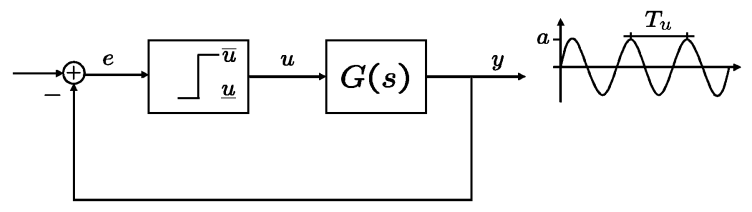

Fig. 1. Relay-feedback experiment.

are the-scalar-transfer functions of the decentralized controller $C(s)$. We deal with PID controllers, in which $p_{i}(s)=k_{p i}+\left(k_{i i} / s\right)+k_{d i} s$, with $k_{p i}, k_{i i}, k_{d i} \in \Re$. Although, in this paper, we focus on PID controllers, the method can be applied to set the parameters of any fixed structure controller.

The outline of this paper is as follows. Section II presents the ultimate-point method for SISO systems, and Section III presents how the ultimate quantities are defined in MIMO processes. The multivariable ultimate-point method is presented in Section IV. As the method needs the knowledge of the process' frequency response at a given frequency, Section $\mathrm{V}$ discusses the practical determination of this quantity. The method is illustrated by two examples: In Section VI, some results of its application to a benchmark - the Wood and Berry distillation column - are given and an additional illustrative example is discussed in Section VII. Finally, Section VIII presents the conclusions.

\section{Ultimate-Point MethoD}

The ultimate-point method for tuning SISO PID controllers is a well-known method based on the knowledge of the ultimate point of the process' frequency response, which is the point at which the process' Nyquist diagram intersects the negative real axis of the complex plane [1]. Associated with this point are the ultimate quantities: the ultimate gain $\left(K_{u}\right)$ and ultimate period $\left(T_{u}\right)$ of the process. If the process is put under purely proportional control, the ultimate gain is the gain for which the loop is at its stability boundary. The ultimate frequency $\left(\omega_{u}\right)$ is the frequency at which the crossing of the negative real axis happens, and the ultimate period is given by $T_{u}=2 \pi / \omega_{u}$. The ultimate quantities are more conveniently obtained by the relay-feedback experiment [1]. This experiment consists of a closed-loop bang-bang control, as shown in Fig. 1. The control function $\eta(\cdot)$ is described as

$$
u=\eta(e)=-d \operatorname{sign}(e)+\operatorname{bias}
$$

where the bias term should be chosen as bias $=r / G(0)$ and the relay amplitude is given by $d=(\underline{u}-\bar{u}) / 2[1]$. Then, if the ultimate point does exist, a symmetric oscillation will be observed, and this oscillation presents the properties as follows.

Fact 1 [1]: Let $\omega_{\text {osc }}$ be the frequency of the oscillation observed in the relay experiment and $a$ be its amplitude at the relay input. Since the ultimate frequency is such that $\angle G\left(\jmath \omega_{u}\right)=-\pi$, $\omega_{\text {osc }}$ gives an estimate for the ultimate frequency, so that both ultimate quantities can be obtained by means of the relay experiment. Then

$$
\omega_{u}=\omega_{\mathrm{osc}}
$$

TABLE I

ZIEGLER-NICHOLS AND TYREUS-LUYBEN FORMULAS

\begin{tabular}{|c||ccc|}
\hline Ziegler-Nichols & $k_{p}$ & $T_{i}$ & $T_{d}$ \\
\hline PI & $0.4 K_{u}$ & $0.8 T_{u}$ & 0 \\
PID & $0.6 K_{u}$ & $0.5 T_{u}$ & $0.125 T_{u}$ \\
\hline \hline Tyreus-Luyben & $k_{p}$ & $T_{i}$ & $T_{d}$ \\
\hline PI & $K_{u} / 3.2$ & $2.2 T_{u}$ & 0 \\
PID & $K_{u} / 2.2$ & $2.2 T_{u}$ & $T_{u} / 6.3$ \\
\hline
\end{tabular}

TABLE II

Different Points to Which the Ultimate PoINT is Moved Using ZIEGLER-NICHOLS AND TYREUS-LUYBEN FORMULAS

\begin{tabular}{|c||c||c|}
\hline Ziegler-Nichols & PI & $-0.4+0.08 \mathrm{\jmath}$ \\
& PID & $-0.6-0.28 \mathrm{\jmath}$ \\
\hline Tyreus-Luyben & PI & $-0.31+0.023 \mathrm{\jmath}$ \\
& PID & $-0.45-0.42 \mathrm{\jmath}$ \\
\hline
\end{tabular}

$$
K_{u}=\frac{4 d}{\pi a} .
$$

Once the ultimate quantities are determined, the PID is tuned according to given formulas. Different sets of tuning rules have been proposed over the years, aiming at different performance criteria. We will explore two sets of formulas in this paper: the originally proposed Ziegler-Nichols formulas [3], which typically provide fast but oscillatory behavior, and the more conservative Tyreus-Luyben formulas [15]. These formulas are given in Table I where the transfer function of the PID controller is given by

$$
C(s)=k_{p}\left(1+\frac{1}{T_{i} s}+T_{d} s\right)
$$

The PID controller dislocates the ultimate point into a stable region, which is away from the point $-1+0 \jmath$ and closer to the origin of the complex plane. Each set of formulas corresponds to moving the ultimate point to a given location in the complex plane. A PI controller with Ziegler-Nichols tuning, for example, has $k_{p}=0.4 K_{u}$ and $\omega_{u} T_{i}=\left(2 \pi / T_{u}\right) 0.8 T_{u}=5.02$. Then, the PI controller transfer function, at the ultimate frequency, is

$$
\begin{aligned}
C\left(\jmath \omega_{u}\right) & =k_{p}\left(1+\frac{1}{\jmath \omega_{u} T_{i}}\right)=0.4 K_{u}\left(1-\frac{\jmath}{5.02}\right) \\
& =K_{u}(0.4-0.08 \mathrm{\jmath})
\end{aligned}
$$

which results in the loop transfer function

$$
L\left(\jmath \omega_{u}\right)=C\left(\jmath \omega_{u}\right) G\left(\jmath \omega_{u}\right)=-0.4+0.08 \jmath .
$$

That is, the ultimate point has been moved to $-0.4+0.08 \mathrm{~J}$. Table II shows the points to which different formulas move a process ultimate point.

\section{A. Stability Margins}

From the Nyquist stability criterion, it follows that if the whole frequency response is kept away from -1 , then the closed-loop system will be stable and present adequate stability (gain and phase) margins. This means that if the ultimate point is away from -1 and closer to the origin, nearby points of the frequency response will also be away and, provided that the 

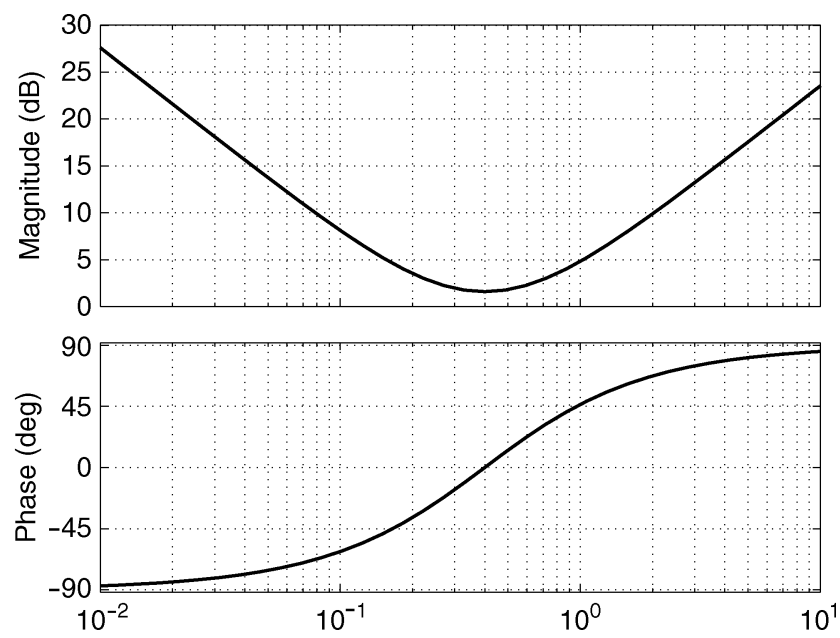

Fig. 2. Bode diagram of a PID controller for a system with $K_{u}=2$ and $\omega_{u}=$ $2 \pi / 10 \mathrm{rad} / \mathrm{s}$.

frequency response is "sufficiently smooth," adequate stability margins are obtained. Different smoothness assumptions on the frequency response have been made in order to associate stability margins to the ultimate-point method [1], [2], [16].

If, for instance, the Ziegler-Nichols tuning is used in a PID controller, then

$$
\begin{aligned}
C(s) & =0.6 K_{u}\left(1+\frac{1}{s \pi / \omega_{u}}+\frac{0.25 \pi s}{\omega_{u}}\right) \\
& =\frac{0.15 \pi K_{u}}{\omega_{u}} \frac{\left(s+\frac{2}{\pi} \omega_{u}\right)^{2}}{s} .
\end{aligned}
$$

That is, the controller presents a double zero at the frequency $-(2 / \pi) \omega_{u}$, at which frequency its magnitude is minimal and equal to $\left|C\left(\jmath(2 / \pi) \omega_{u}\right)\right|=0.6 K_{u}$. Hence, the frequency response of the controller has the form shown in Fig. 2.

Stability in closed loop is obtained if the loop transfer function $C(\jmath \omega) G(\jmath \omega)$ satisfies

$$
\begin{aligned}
& \left|C\left(\jmath \omega_{1}\right) G\left(\jmath \omega_{1}\right)\right|<1 \\
& \angle C\left(\jmath \omega_{0}\right) G\left(\jmath \omega_{0}\right) \mid \geq-180^{\circ}
\end{aligned}
$$

where $\omega_{1}$ and $\omega_{0}$ are defined as $\angle C\left(\jmath \omega_{1}\right) G\left(\jmath \omega_{1}\right) \mid=-\pi$ and $\left|C\left(\jmath \omega_{0}\right) G\left(\jmath \omega_{0}\right)\right|=1$, respectively. With the Ziegler-Nichols tuning, we have by definition $C\left(\jmath \omega_{u}\right) G\left(\jmath \omega_{u}\right)=-0.6-0.28 \jmath=$ $0.66 \angle-25.02^{\circ}$. If the process' module drops faster than the growth of the controller's module for all frequencies above $\omega_{u}$ and its phase drops faster than the growth of the controller's phase for all frequencies below $\omega_{u}$, then it is clear that conditions (8) and (9) will be satisfied. We have thus established the following fact.

Fact 2: Let $G(s)$ be the transfer function of a BIBO-stable SISO process, and let this process be controlled by a PID controller (6) with the Ziegler-Nichols tuning as given in Table I. If the Bode plot of the process' frequency response lies completely outside the shaded area as shown in Fig. 3, then the closed-loop system is BIBO stable.

The constraints on the frequency response of the process in the fact above have clear physical interpretation. Although

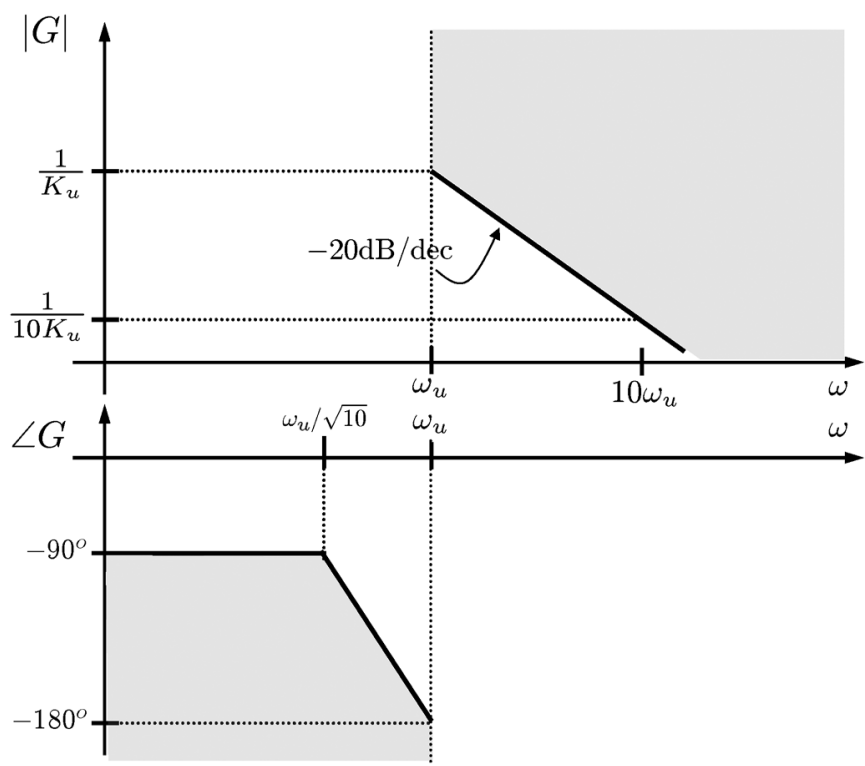

Fig. 3. Stability boundaries for the process' frequency response.

these conditions can usually not be directly checked for a given process (checking them would require a rough model of the process), they give a guideline to the class of processes to which Ziegler-Nichols tuning can be successfully applied. For instance, a delay-free stable transfer function with no zeros will always satisfy these constraints provided that its poles are not too far apart from each other. On the other hand, several classes of processes are excluded, such as systems with dominant time-delay and oscillatory systems with natural oscillation frequency close to the ultimate frequency. For these systems, stability cannot be guaranteed, and for many among them, the closed-loop system will indeed be unstable if a Ziegler-Nichols tuning is applied.

Different sufficient conditions, with different degrees of conservatism, can be obtained for the Ziegler-Nichols tuning, as well as for other tuning rules [1], [16]. The same considerations will apply regarding the closed-loop stability when applying the multivariable ultimate-point method.

\section{Ultimate Quantities In Mimo Processes}

In multivariable processes like (1), the ultimate quantities can be defined similarly to the SISO case [14].

Definition 1: Let a BIBO-stable square process with $m$ inputs be controlled by purely proportional controller $u=-K y$, with gain $K=\operatorname{diag}\left\{k_{1} k_{2}, \ldots, k_{m}\right\}, k_{i} \in[0, \infty)$. Since the process is BIBO stable, the feedback system is BIBO stable for sufficiently small $K$. Assume that there exists a constant matrix $K_{u}$ such that the closed-loop system is BIBO stable $\forall K=\alpha K_{u}, 0<\alpha<1$, and unstable for $K=K_{u}(1+\epsilon)$, with $\epsilon$ an arbitrarily small positive scalar; this value $K_{u}$ is called an ultimate gain of the process. On the other hand, for $K=K_{u}$, the closed-loop system is on the verge of stability, and hence, a sustained oscillation will be observed; the frequency of this oscillation is called an ultimate frequency $\omega_{u}$.

The stability of MIMO square systems can be analyzed through the Nyquist criterion, as in the SISO case. Denote 


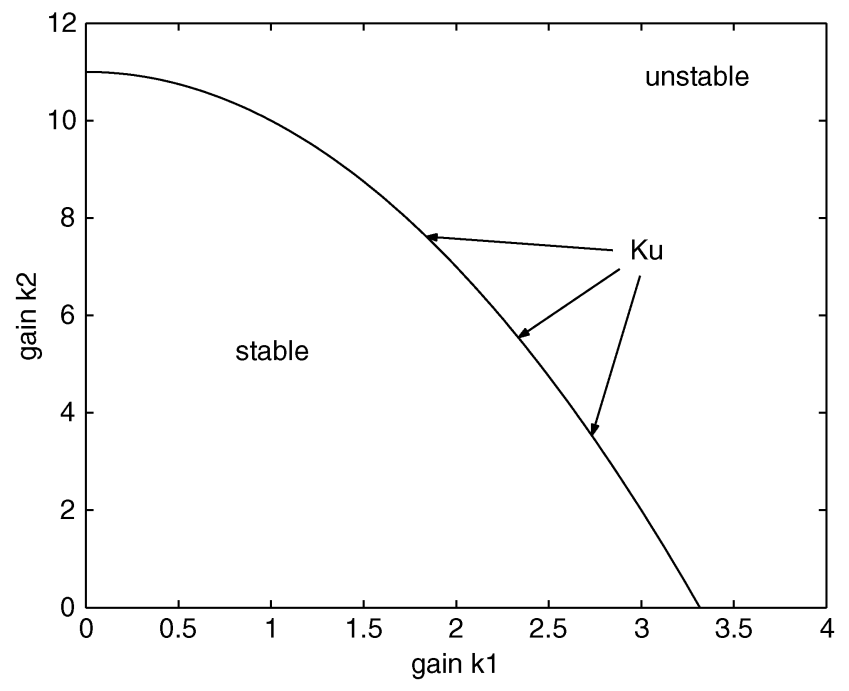

Fig. 4. Ultimate gains in the TITO case form a curve in the parameter space.

$\lambda_{i}(s), i=1,2, \ldots, m$, the eigenvalues of $G(s) K$. The graphs of $\lambda_{i}(s)$ as $s$ goes round the Nyquist contour are called the characteristic loci. With these definition, the Nyquist theorem for MIMO systems can be stated as follows.

Theorem 1 (Generalized Nyquist Theorem) [8]: If $G(s)$ has $P_{0}$ unstable (Smith-McMillan) poles, then the closed-loop system with return ratio $-G(s) K$ is stable if and only if the characteristic loci of $G(s) K$, taken together, encircle the point $-1 P_{0}$ times counterclockwise, assuming that there are no hidden unstable modes.

Therefore, for a BIBO stable $G(s)$, the closed-loop system is stable if and only if none of the characteristic loci of $G(s) K$ encircles the point -1 . As the gain matrix $K$ is varied, the stability limit is reached when at least one of the characteristic loci equals -1 for some frequency. The gain for which this happens is an ultimate gain.

The ultimate quantities are usually unique in the SISO case; even when they are not unique, they are countable. The situation is quite different in the MIMO case, since the gain matrix $K$ can be increased from zero in infinite different directions in the parameter space. It can be expected that a different $K_{u}$ and $\omega_{u}$ will be found for each different direction, as shown in Fig. 4. The set of all the ultimate gains is a curve in the parameter space. In the more general $(m>2)$ case, these gains will form a surface of dimension $m-1$; this surface will be called the ultimate surface[14].

If decentralized PID tuning is determined based on the ultimate quantities using Ziegler-Nichols-like formulas, as in [10]-[12], and [17], then two things must be realized. First, all PIDs will be tuned based on the same ultimate frequency. Second, the tuning will be dependent on which pair of the ultimate quantities has been identified.

For simplicity, let $m=2$. Consider that the gain $K$ is increased in the direction $K=\operatorname{diag}\left\{k_{1} 0\right\}$, i.e., the second loop is kept open, and the proportional gain in loop one is increased. The ultimate gain that will be obtained in this case is the SISO

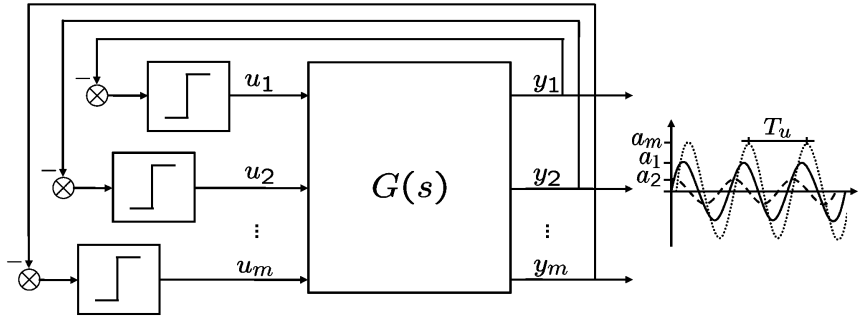

Fig. 5. DRF experiment.

ultimate gain $k_{u 1}$ of the first loop. Then, if the PID is tuned according to these ultimate quantities, then this tuning is the "correct" one for the first SISO loop. On the other hand, if the gain $K$ is increased as $K=\operatorname{diag}\left\{0 k_{2}\right\}$, then the "correct" SISO tuning for the second loop is obtained. If a different direction in the parameter space is picked to increase the gain $K$, then it is expected to find a tuning that will not be optimal for any of the two loops but will represent some sort of "average" of the two. The closer this direction is to either one of the two SISO directions defined earlier, the closer the tuning is expected to be to the corresponding "correct" SISO tuning for that loop [10].

Once the ultimate quantities are identified, a gain sufficiently smaller than the ultimate one should guarantee stability and enough stability margins. However, this is not necessarily true in the MIMO case. If the ultimate curve in the parameter space is convex, then adequate stability margins are guaranteed by taking a gain $K=\alpha K_{u}$ for sufficiently small $\alpha$. However, it is not possible to guarantee that the ultimate surface is convex or even smooth. As a matter of fact, there are cases in which the ultimate surface is not convex, like in the benchmark studied in Section VI.

The ultimate gains can be more conveniently obtained by a decentralized relay-feedback (DRF) experiment [10], shown in Fig. 5, and different ultimate points are identified with different relay amplitudes [13], [14]. In DRF, only one experiment is performed, with all control loops in relay feedback, i.e., $u_{i}=\eta\left(e_{i}\right) \forall i$. Since all the input-output pairs are connected, the behavior of the whole multivariable system is observed in this single experiment.

\section{Design Based on the Mimo ULTiMATE QUANTITIES}

The multivariable ultimate-point method is an extension of the ultimate-point method to multivariable systems. The main idea is to dislocate the ultimate point of the process to another point in the complex plane, moving it to a position that is away from the point $-1+0 \jmath$ and closer to the origin [13]. Let us first present the two-input-two-output (TITO) case.

\section{A. TITO Case}

Consider a $2 \times 2$ or TITO system. Then, the loop transfer function for $K=K_{u}$ is given by

$$
G(\jmath \omega) K_{u}=\left[\begin{array}{ll}
g_{11}(\jmath \omega) & g_{12}(\jmath \omega) \\
g_{21}(\jmath \omega) & g_{22}(\jmath \omega)
\end{array}\right]\left[\begin{array}{cc}
k_{u 1} & 0 \\
0 & k_{u 2}
\end{array}\right] .
$$

Let $\lambda_{1}(\jmath \omega)$ and $\lambda_{2}(\jmath \omega)$ be the eigenvalues of the loop transfer matrix $G(\jmath \omega) K_{u}$. Then, by the very definition of critical gain 
$K_{u}$ and critical frequency $\omega_{u}$, at least one of these eigenvalues is equal to -1 for $\omega=\omega_{u}$, i.e., either $\lambda_{1}\left(\jmath \omega_{u}\right)=-1$ or $\lambda_{2}\left(\jmath \omega_{u}\right)=-1$ or both. Replacing the proportional controller with a decentralized dynamic controller in (10), we have

$$
G(\jmath \omega) C(\jmath \omega)=\left[\begin{array}{ll}
g_{11}(\jmath \omega) & g_{12}(\jmath \omega) \\
g_{21}(\jmath \omega) & g_{22}(\jmath \omega)
\end{array}\right]\left[\begin{array}{cc}
p_{1}(\jmath \omega) & 0 \\
0 & p_{2}(\jmath \omega)
\end{array}\right] .
$$

The ultimate point is to be dislocated to a previously chosen position in the complex plane, i.e., at the ultimate frequency $\omega_{u}$; the eigenvalues of the loop matrix in (11) must be located at these previously chosen locations. From (11), the characteristic loci at the ultimate frequency are given by

$$
\operatorname{det}\left(\lambda\left(\jmath \omega_{u}\right) I-G\left(\jmath \omega_{u}\right) C\left(\jmath \omega_{u}\right)\right)=0 .
$$

The ultimate-point design consists in finding the controller's transfer functions $p_{1}(s)$ and $p_{2}(s)$ such that the eigenvalues $\lambda\left(\jmath \omega_{u}\right)$ in (12) have the desired values, for example, $\Lambda_{1}$ and $\Lambda_{2}$; this is the core aspect of the MIMO ultimate-point method. Let us give a more detailed treatment for a particular way of choosing these locations in the complex plane, namely, $\Lambda_{1}=\Lambda_{2}=\Lambda$. With this choice, expanding (12) and defining $g_{11_{u}}=g_{11}\left(\jmath \omega_{u}\right), g_{12_{u}}=g_{12}\left(\jmath \omega_{u}\right), g_{21_{u}}=g_{21}\left(\jmath \omega_{u}\right)$, $g_{22_{u}}=g_{22}\left(\jmath \omega_{u}\right), p_{1_{u}}=p_{1}\left(\jmath \omega_{u}\right)$, and $p_{2_{u}}=p_{2}\left(\jmath \omega_{u}\right)$ in order to simplify the notation, we have

$\lambda^{2}-\left(g_{11_{u}} p_{1_{u}}+g_{22_{u}} p_{2_{u}}\right) \lambda+\left(g_{11_{u}} g_{22_{u}}-g_{12_{u}} g_{21_{u}}\right) p_{1_{u}} p_{2_{u}}=0$.

If the roots of (13) are given by $\Lambda$, then the characteristic equation is given by $\lambda^{2}-2 \Lambda \lambda+\Lambda^{2}$, which yields

$$
\begin{aligned}
& 2 \Lambda=g_{11_{u}} p_{1_{u}}+g_{22_{u}} p_{2_{u}} \\
& \Lambda^{2}=\left(g_{11_{u}} g_{22_{u}}-g_{12_{u}} g_{21_{u}}\right) p_{1_{u}} p_{2_{u}} .
\end{aligned}
$$

From (14), we have

$$
p_{2_{u}}=\frac{2 \Lambda}{g_{22_{u}}}-\frac{g_{11_{u}}}{g_{22_{u}}} p_{1_{u}}
$$

and substituting (16) into (15), we have

$$
\frac{g_{11_{u}}}{g_{22_{u}}} p_{1_{u}}^{2}-\frac{2 \Lambda}{g_{22_{u}}} p_{1_{u}}+\frac{\Lambda^{2}}{g_{11_{u}} g_{22_{u}}-g_{12_{u}} g_{21_{u}}}=0 .
$$

Since (17) is a second-order polynomial, we can get two different tunings for $p_{1_{u}}$. Note that $p_{1_{u}}$ is a complex quantity. Then, by solving (17), we have the setting of the tunings for $p_{1_{u}}$, and, substituting into (16), two different tunings for $p_{2_{u}}$, also complex, are obtained. Therefore, we have two different controllers that move the ultimate point to the desired location.

For PI controllers, from the solution of (17), we have

$$
\begin{aligned}
k_{p 1} & =\operatorname{Re}\left\{p_{1_{u}}\right\} \\
k_{i 1} & =-\operatorname{Im}\left\{p_{1_{u}}\right\} \omega_{u}
\end{aligned}
$$

and $p_{2_{u}}$ gains are given by

$$
k_{p 2}=\operatorname{Re}\left\{p_{2_{u}}\right\}
$$

$$
k_{i 2}=-\operatorname{Im}\left\{p_{2_{u}}\right\} \omega_{u} .
$$

For PID controllers, we obtain $p_{1_{u}}$ in the same way [from (17)], but in this case

$$
p_{1_{u}}=k_{p 1}\left(1+\left(T_{d 1} \omega_{u}-\frac{1}{T_{i 1} \omega_{u}}\right) \jmath\right)
$$

and we have

$$
\begin{aligned}
k_{p_{1}} & =\operatorname{Re}\left\{p_{1_{u}}\right\} \\
k_{p_{1}}\left(T_{d 1} \omega_{u}-\frac{1}{T_{i 1} \omega_{u}}\right) & =\operatorname{Im}\left\{p_{1_{u}}\right\} .
\end{aligned}
$$

A 1 DOF still exists in the choice of $T_{i 1}$ and $T_{d 1}$. A possible choice is to set $T_{d 1}=T_{i 1} / 4$, which is usual also in SISO design, causing the two zeros of the controller to be equal, which guarantees smoothness of the controller's frequency response. In this case, (23) becomes

$$
k_{p_{1}}\left(T_{i 1} \omega_{u}\right)^{2}-4 \operatorname{Im}\left\{p_{1_{u}}\right\} T_{i 1} \omega_{u}-4 k_{p 1}=0 .
$$

Thus, from (24), we have two solutions for $T_{i 1} \omega_{u}$, which are always real and with opposite signs. We choose the solution where $T_{i 1} \omega_{u}>0$, and from the relations $k_{i 1}=k_{p 1} / T_{i 1}$ and $k_{d 1}=k_{p 1} T_{i 1} / 4$, we obtain the gains of the controller $p_{1_{u}}$. Controller $p_{2_{u}}$ is obtained from (16), and then, its parameters $T_{i 2}$ and $k_{p 2}$ from (24)mutatis mutandis. As for PI controllers, we find two different PID controllers that dislocate both characteristic loci to the desired point.

\section{B. General Case}

For the general MIMO case, where $m$ may be different from two, the same reasoning holds and the same procedure can be applied. Of course, the equations become more complex. Consider again the case in which all characteristic loci are dislocated to the same desired point $\Lambda$; for a TITO process, the controllers are obtained through (14) and (15). For a process of order $m$, the equations used to obtain the controllers are given by

$$
a_{m-i} \Lambda^{i}=\sum M_{i}\left(G\left(j \omega_{u}\right) C\left(j \omega_{u}\right)\right)
$$

where $M_{i}$ are the $i$ th-order principal minors of the matrix $G\left(j \omega_{u}\right) C\left(j \omega_{u}\right)$, and each sum is taken over all principal minors of the order $i$. We note that, in each term, there are always $\mathcal{C}_{m}^{i}=m ! / i !(m-i) !$ such minors, where $\mathcal{C}_{m}^{i}$ stands for the combination of $m$ elements taken $i$ at a time.

On the other hand, $a_{m-i}$ are the coefficients of the characteristic equation $(s-\lambda)^{m}$, which can be obtained using the Newton's binomial formula, for $i=1, \ldots, m$. Therefore, we have

$$
a_{m-i}=\mathcal{C}_{m}^{i}=\frac{m !}{(m-i) ! i !} .
$$

Like in the TITO case, this will result in a system of equations with the controllers' values as unknowns. 
We present the case where $m=3$ for illustration. The coefficients of the characteristic equation are given by (26)

$$
\left\{\begin{array}{l}
i=1 \rightarrow a_{2} \Lambda=\mathcal{C}_{3}^{1} \Lambda=3 \Lambda \\
i=2 \rightarrow a_{1} \Lambda^{2}=\mathcal{C}_{3}^{2} \Lambda^{2}=3 \Lambda^{2} \\
i=3 \rightarrow a_{0} \Lambda^{3}=\mathcal{C}_{3}^{3} \Lambda^{3}=\Lambda^{3}
\end{array}\right.
$$

Equating (27) with (25) results in the following set of equations:

$$
\begin{aligned}
3 \Lambda= & g_{11_{u}} p_{1_{u}}+g_{22_{u}} p_{2_{u}}+g_{33_{u}} p_{3_{u}} \\
3 \Lambda^{2}= & \left(g_{11_{u}} g_{22_{u}}-g_{12_{u}} g_{21_{u}}\right) p_{1_{u}} p_{2_{u}} \\
& +\left(g_{11_{u}} g_{33_{u}}-g_{13_{u}} g_{31_{u}}\right) p_{1_{u}} p_{3_{u}} \\
& +\left(g_{22_{u}} g_{33_{u}}-g_{23_{u}} g_{32_{u}}\right) p_{2_{u}} p_{3_{u}} \\
\Lambda^{3}= & {\left[\left(g_{11_{u}} g_{22_{u}} g_{33_{u}}+g_{21_{u}} g_{13_{u}} g_{32_{u}}+g_{31_{u}} g_{12_{u}} g_{23_{u}}\right)\right.} \\
& \left.-\left(g_{11_{u}} g_{23_{u}} g_{32_{u}}+g_{21_{u}} g_{12_{u}} g_{33_{u}}+g_{31_{u}} g_{13_{u}} g_{22_{u}}\right)\right] \\
& \times p_{1_{u}} p_{2_{u}} p_{3_{u}} .
\end{aligned}
$$

The controller is obtained by solving (28), (29), and (30) for the unknowns $p_{1 u}, p_{2 u}$, and $p_{3 u}$. This results in six different solutions - in contrast to the two solutions for the TITO case-and it is not clear how to choose among them. Other design choices also become significantly more involved for systems of dimension larger than two. The handling of these choices is by no means straightforward, so this is a topic that should be further developed to make our method more practical for systems with more than two inputs, although it is, in principle, applicable to systems of any dimension.

\section{Practical Determination of $G\left(\jmath \omega_{u}\right)$}

In the SISO ultimate-point method, the design of the controller requires the knowledge of the process' frequency response at the ultimate point, an information that is provided by the relay-feedback experiment. Accordingly, the MIMO ultimate-point method, as presented in the previous section, requires knowledge of the transfer matrix at the ultimate frequency. However, the DRF does not provide the whole transfer matrix but only the ultimate quantities of the process, which for MIMO processes is not equivalent. There are different ways of determining $G\left(\jmath \omega_{u}\right)$, and the more accurate ones will be more demanding. The aim of this section is to present a convenient procedure to apply in operating systems, although the development of optimized procedures remains an open issue.

Consider a TITO process. When the DRF is applied, $u_{1}(t)$, $u_{2}(t), y_{1}(t)$, and $y_{2}(t)$ are periodic signals. Let $u_{1}, u_{2}, y_{1}$, and $y_{2}$ be the complex coefficients of the first harmonics of these signals. Then, we have

$$
\left\{\begin{array}{l}
y_{1}=g_{11}\left(\jmath \omega_{u}\right) u_{1}+g_{12}\left(\jmath \omega_{u}\right) u_{2} \\
y_{2}=g_{21}\left(\jmath \omega_{u}\right) u_{1}+g_{22}\left(\jmath \omega_{u}\right) u_{2}
\end{array}\right.
$$

As we have two equations and four unknowns, it is not possible to determine $G\left(\jmath \omega_{u}\right)$. Therefore, an additional experiment is necessary. From the DRF test, we know the ultimate frequency $\omega_{u}$ of the system. Therefore, if we apply an input $u_{1}(t)=$ $\sin \left(\omega_{u} t\right)$ and $u_{2}(t)=0$ to the system, we have

$$
\left\{\begin{array}{l}
y_{1}=g_{11}\left(\jmath \omega_{u}\right) u_{1} \\
y_{2}=g_{21}\left(\jmath \omega_{u}\right) u_{1}
\end{array}\right.
$$

and it is easy to determine $g_{11}\left(\jmath \omega_{u}\right)$ and $g_{21}\left(\jmath \omega_{u}\right)$. Substituting these quantities in (31), we determine $G\left(\jmath \omega_{u}\right)$. However, this additional experiment is an open-loop test, which is usually inconvenient in real applications. In many situations, it is difficult to apply this signal to the input of the process depending on the software and hardware structure in the control system implementation.

A more convenient solution is to apply a second DRF test on the system, changing one relay's amplitude in such a way that the oscillation frequency does not change significantly. Thus, we have

$$
\left\{\begin{array}{l}
y_{1}^{I}=g_{11}\left(\jmath \omega_{u}^{I}\right) u_{1}^{I}+g_{12}\left(\jmath \omega_{u}^{I}\right) u_{2}^{I} \\
y_{2}^{I}=g_{21}\left(\jmath \omega_{u}^{I}\right) u_{1}^{I}+g_{22}\left(\jmath \omega_{u}^{I}\right) u_{2}^{I} \\
y_{1}^{I I}=g_{11}\left(\jmath \omega_{u}^{I I}\right) u_{1}^{I I}+g_{12}\left(\jmath \omega_{u}^{I I}\right) u_{2}^{I I} \\
y_{2}^{I I}=g_{21}\left(\jmath \omega_{u}^{I I}\right) u_{1}^{I I}+g_{22}\left(\jmath \omega_{u}^{I I}\right) u_{2}^{I I}
\end{array}\right.
$$

where the indexes $I$ and $I I$ mean the first and the second DRF tests in the plant and the two oscillating frequencies $\omega_{u}^{I}$ and $\omega_{u}^{I I}$ are close to each other. If we make the approximation $\omega_{u}^{I}=\omega_{u}^{I I}$, we have four equations and four unknowns, and we can estimate $\hat{G}\left(\jmath \omega_{u}\right)$ by

$$
\begin{aligned}
{\left[\begin{array}{ll}
\hat{g}_{11} & \hat{g}_{12} \\
\hat{g}_{21} & \hat{g}_{22}
\end{array}\right]\left[\begin{array}{ll}
u_{1}^{I} & u_{1}^{I I} \\
u_{2}^{I} & u_{2}^{I I}
\end{array}\right] } & =\left[\begin{array}{ll}
y_{1}^{I} & y_{1}^{I I} \\
y_{2}^{I} & y_{2}^{I I}
\end{array}\right] \\
\hat{G} U & =Y \\
\hat{G} & =Y U^{-1} .
\end{aligned}
$$

Having these information, the multivariable ultimate-point method can be used in order to obtain the controller. The difference between the two frequencies will introduce an error in the estimation of $G\left(\jmath \omega_{u}\right)$, but as the oscillation frequencies are quite similar, we can expect this error to be small [16]. The case studies presented in the sequel show that this is indeed the case and that this procedure can, in many cases, be used to obtain the matrix $G\left(\jmath \omega_{u}\right)$.

\section{CASE Study-Distillation COLUMN}

Aiming at demonstrating the use of the proposed design method, this section presents some results obtained for the control of the Wood and Berry distillation column, whose transfer matrix is presented in (35). This process has been widely used as a benchmark [11], [18]

$$
G(s)=\left[\begin{array}{cc}
\frac{12.8 e^{-s}}{16.7 s+1} & \frac{-18.9 e^{-3 s}}{21 s+1} \\
\frac{6.6 e^{-7 s}}{10.9 s+1} & \frac{-19.4 e^{-3 s}}{14.4 s+1}
\end{array}\right] .
$$

Since the process is $2 \times 2$, we have infinite different combinations of $k_{u 1}$ and $k_{u 2}$ that lead the process to the stability limit, each one corresponding to a different ultimate point. 
TABLE III

$\hat{G}\left(\jmath \omega_{u}\right)$ FOR Two DifFerent FreQUENCIES OF WOOD AND BERRY COLUMN

\begin{tabular}{|c|cc|c|cc|}
\hline experiment & $\left|u_{2}\right| /\left|u_{1}\right|$ & $\omega_{u}$ & $\hat{G}\left(\jmath \omega_{u}\right)$ & $\Delta G$ & $e(\%)$ \\
\hline 1 & 1.9371 & 0.4946 & $\hat{G}_{1}=\left[\begin{array}{cc}-0.5554-1.4295 \jmath & 1.7820+0.3223 \jmath \\
0.1784+1.1898 \jmath & 2.6252+0.5941 \jmath\end{array}\right]$ & 2.2 & 0.6 \\
& 0.9489 & 0.4931 & & & \\
\hline 3 & 3.5303 & 0.5228 \\
4 & 0.6659 & 0.5106 & $\hat{G}_{2}=\left[\begin{array}{ccc}-0.5843-1.3663 \jmath & 1.7265+0.2054 \jmath \\
0.2731+1.1438 \jmath & 2.5441+0.4183 \jmath\end{array}\right]$ & 7.0 & 6.6 \\
\hline
\end{tabular}

In order to illustrate the results, we have tested different ultimate points. As the multivariable ultimate-point method needs the knowledge of $G\left(\jmath \omega_{u}\right)$, we have to find two different but very close ultimate points and then estimate $\hat{G}\left(\jmath \omega_{u}\right)$ by (34). We have estimated $\hat{G}\left(\jmath \omega_{u}\right)$ for two different frequencies, which are presented in Table III, where

$$
\Delta G=\frac{\left\|G\left(\jmath \omega_{u}^{I}\right)-G\left(\jmath \omega_{u}^{I I}\right)\right\|_{2}}{\left\|G\left(\jmath \omega_{u}^{I}\right)\right\|_{2}}
$$

is the variation of $G$ due to the difference of the oscillation frequencies obtained in each DRF test and

$$
e=\frac{\left\|G\left(\jmath \omega_{u}^{I}\right)-\hat{G}\left(\jmath \omega_{u}^{I}\right)\right\|_{2}}{\left\|G\left(\jmath \omega_{u}^{I}\right)\right\|_{2}}
$$

is the estimation error of $\hat{G}\left(\jmath \omega_{u}\right)$ related to $G\left(\jmath \omega_{u}\right) ; \omega_{u}$ is obtained from the first DRF test performed.

\section{A. PID Controller Obtained Using the Ziegler-Nichols Formulas}

This section presents some results obtained for the Wood and Berry distillation column using the ultimate quantities of the process and Ziegler-Nichols formulas. This tuning procedure is adopted in several papers [10], [11], [18]. We show the results for references $r(t)=\left[\begin{array}{ll}1 & 0\end{array}\right]^{\prime}$ and $r(t)=\left[\begin{array}{ll}0 & 1\end{array}\right]^{\prime}$.

Fig. 6 shows the behavior of the system with a PID controller tuned through the Ziegler-Nichols formulas and the ultimate quantities $K_{u}=[0.42-0.30]$ and $\omega_{u}=0.4882 \mathrm{rad} / \mathrm{s}$. Note that the transient performance obtained is very good. However, for a PID controller tuned based on another point of the ultimate surface, $K_{u}=[1.27-0.25]$ and $\omega_{u}=0.5129 \mathrm{rad} / \mathrm{s}$, the system became unstable, as shown in Fig. 7. These results show that the SISO tuning is too risky, due to the nonexistence of a full multivariable analysis to justify the tuning procedure.

\section{B. PID Controller Obtained With Multivariable Ultimate-Point Method}

Next, we have found a PID controller that moves the characteristic loci to the point $-0.45-0.42 \mathrm{\jmath}$, which is the equivalent of Tyreus-Luyben point for the SISO ultimate-point method. As (23) yields two solutions, Fig. 8 shows the characteristic loci of the system with each one of the controllers inserted.

The outermost characteristic loci of both systems-system controlled by PID 1 and system controlled by PID 2-cross the circle of unitary radius approximately at the same point. At every point of this circle, the magnitude of the system's response
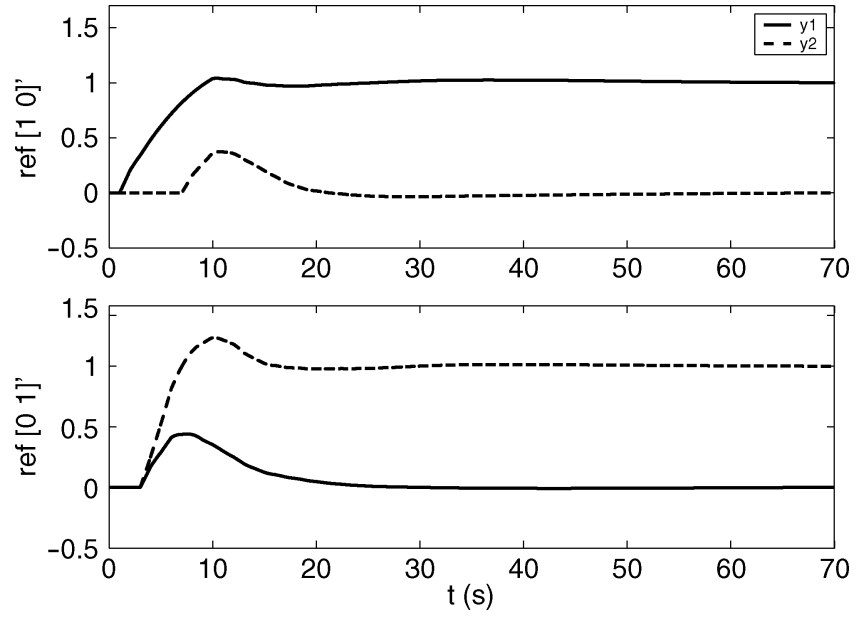

Fig. 6. Step response of the system with PID tuned using Ziegler-Nichols formulas and ultimate point $K_{u}=[0.42-0.30]$ and $\omega_{u}=0.4882 \mathrm{rad} / \mathrm{s}$.
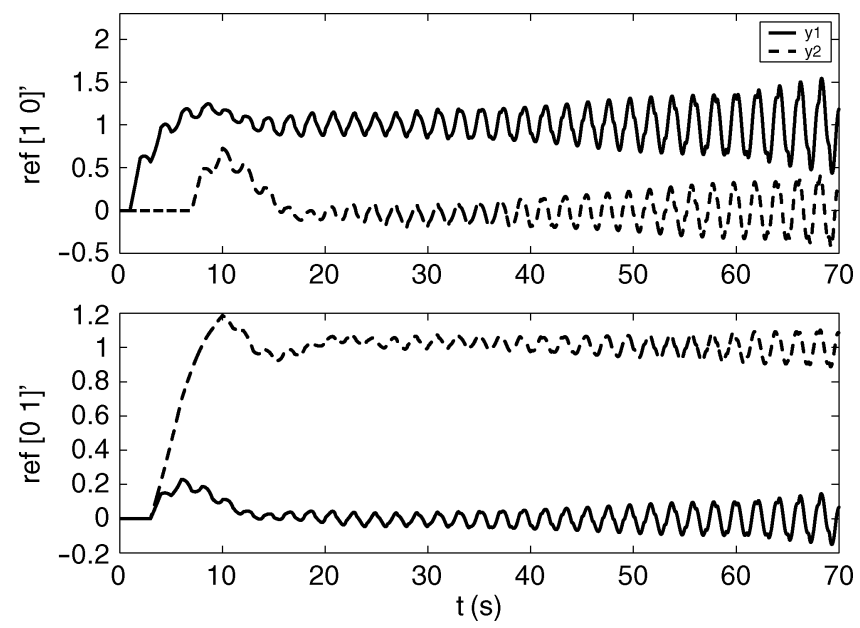

Fig. 7. Step response of the system with PID tuned using Ziegler-Nichols formulas and ultimate point $K_{u}=[1.27-0.25]$ and $\omega_{u}=0.5129 \mathrm{rad} / \mathrm{s}$.

is equal to one, and the phase margin is given by the angle between the crossing of the outermost characteristic locus with the circle of unitary radius and the negative real axis. This means that the phase margin of both systems is the same. Table IV presents the controllers' gains and the stability margins, and Fig. 9 shows both systems' responses for different references. As can be seen in Table IV, the phase margins are almost the same for the two controllers-close to $33^{\circ}$-but PID 2 presents a worse gain margin. The effect of this fact can be seen in the step responses of the systems shown in Fig. 9, in which $y_{2}(t)$ of 
TABLE IV

PID CONTROLLERS' GAINS OF THE SySTEMS SHOWn IN Fig. 9

\begin{tabular}{|c|cccccccc|}
\hline Controller & $k_{p 1}$ & $k_{i 1}$ & $k_{d 1}$ & $k_{p 2}$ & $k_{i 2}$ & $k_{d 2}$ & $G_{M}$ & $\Phi_{M}$ \\
\hline PID 1 & 0.5823 & 0.2247 & 0.3773 & -0.0860 & -0.0135 & -0.1374 & $2.57 I$ & $33.25^{\circ}$ \\
PID 2 & 0.1435 & 0.0623 & 0.0826 & -0.3123 & -0.0434 & -0.5617 & $1.23 I$ & $32.59^{\circ}$ \\
\hline
\end{tabular}

TABLE V

PID Controllers' Gains That Dislocate the Ultimate Point to-0.2 - $0.3 \mathrm{\jmath}$

\begin{tabular}{|cc|cccccccc|}
\hline$\hat{G}$ & Controller & $k_{p 1}$ & $k_{i 1}$ & $k_{d 1}$ & $k_{p 2}$ & $k_{i 2}$ & $k_{d 2}$ & $G_{M}$ & $\Phi_{M}$ \\
\hline 1 & PID 1 & 0.3663 & 0.1102 & 0.3044 & -0.0432 & -0.0051 & -0.0912 & $3.8760 I$ & $42.50^{\circ}$ \\
1 & PID 2 & 0.0929 & 0.0311 & 0.0693 & -0.1510 & -0.0155 & -0.3671 & $1.8975 I$ & $45.78^{\circ}$ \\
\hline 2 & PID 1 & 0.3803 & 0.1149 & 0.3147 & -0.0416 & -0.0045 & -0.0952 & $3.2457 I$ & $43.05^{\circ}$ \\
2 & PID 2 & 0.0968 & 0.0315 & 0.0742 & -0.1493 & -0.0147 & -0.3796 & $1.8325 I$ & $47.09^{\circ}$ \\
\hline
\end{tabular}

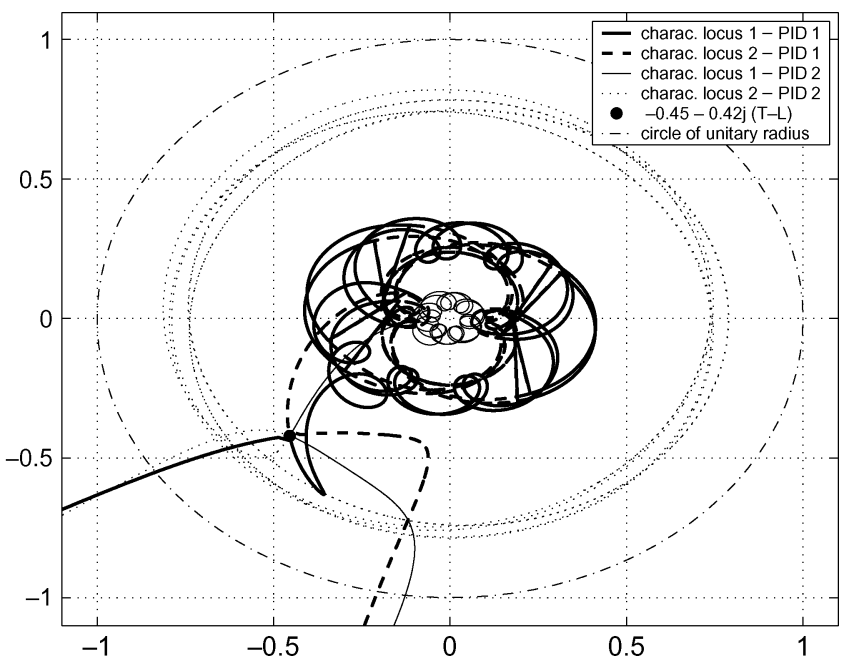

Fig. 8. Characteristic loci of the distillation column with both PID controllers, designed with the proposed method.
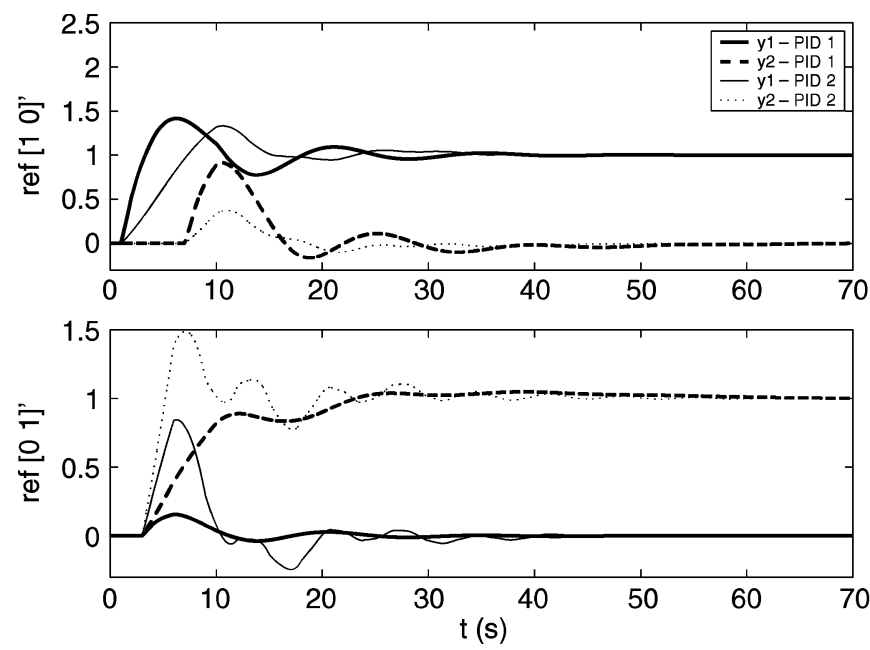

Fig. 9. Step responses of the system with PID 1 and PID 2, obtained based on Tyreus-Luyben point.

the system controlled by PID 2 presents a significant overshoot for reference $r=\left[\begin{array}{ll}0 & 1\end{array}\right]^{\prime}$. Fig. 9 shows the step responses of the system with the controllers obtained with $\hat{G}_{1}\left(\jmath \omega_{u}\right)$.

The performance of the system can be improved through the choice of another point in the complex plane, more conservative
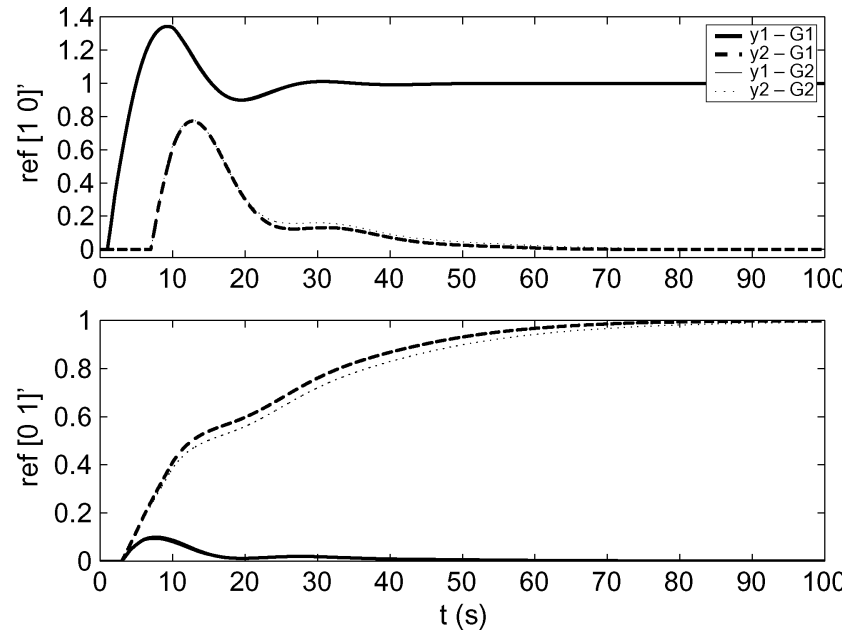

Fig. 10. Step response of the system with PID 1 controller that dislocates the ultimate point to $-0.2-0.3 \mathrm{~J}$

than Ziegler-Nichols and Tyreus-Luyben ones. Several controllers were designed for different points in the complex plane $\left(\Lambda^{\prime} s\right)$, and Figs. 10 and 11 show the step responses of the system with PID 1 and 2, respectively, that dislocate the ultimate point to $-0.2-0.3 \mathrm{j}$. Table $\mathrm{V}$ presents the controllers' gains and stability margins. In this case, we present the controllers obtained with $\hat{G}_{1}\left(\jmath \omega_{u}\right)$ and $\hat{G}_{2}\left(\jmath \omega_{u}\right)$, which correspond to two different ultimate points. Different controllers were designed with different ultimate points, and all these controllers yield similar results.

The system performances corresponding to $\hat{G}_{1}\left(\jmath \omega_{u}\right)$ and $\hat{G}_{2}\left(\jmath \omega_{u}\right)$ are not visually distinguishable, reinforcing the fact that the choice of the "right" ultimate point loses importance when the MIMO ultimate-point method is applied. This is also verified by the controllers' gains obtained for different ultimate points, which are very similar.

In addition, as the point in the complex plane chosen is more conservative than the one used before, there is a significant increase in the stability margins; step responses just validate that.

\section{PI Controller Obtained With Multivariable Ultimate-Point Method for One Characteristic Locus}

Let us present a simpler alternative to the previous choice of dislocation for the ultimate point. The stability limit is usually 

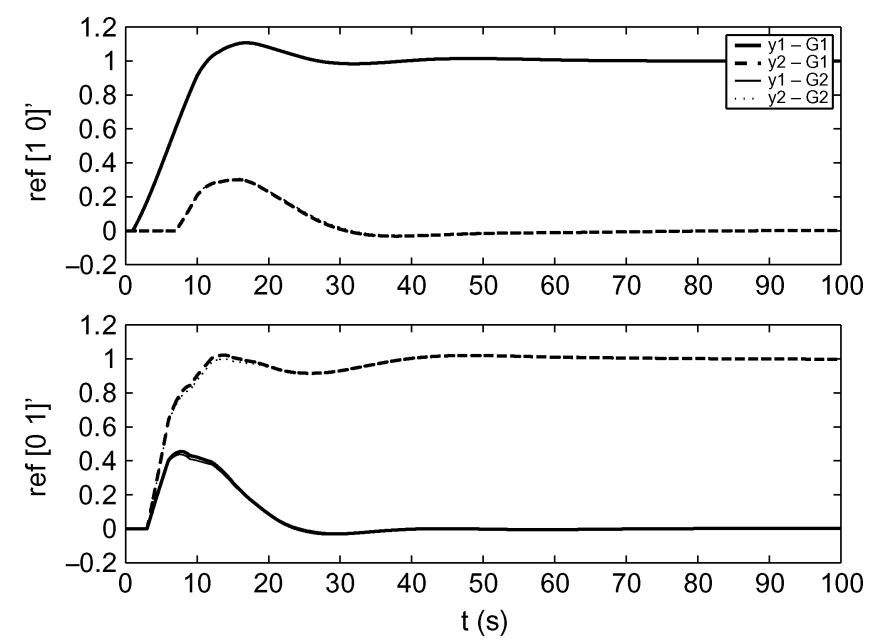

Fig. 11. Step response of the system with PID 2 controller that dislocates the ultimate point to $-0.2-0.3 \mathrm{~J}$.

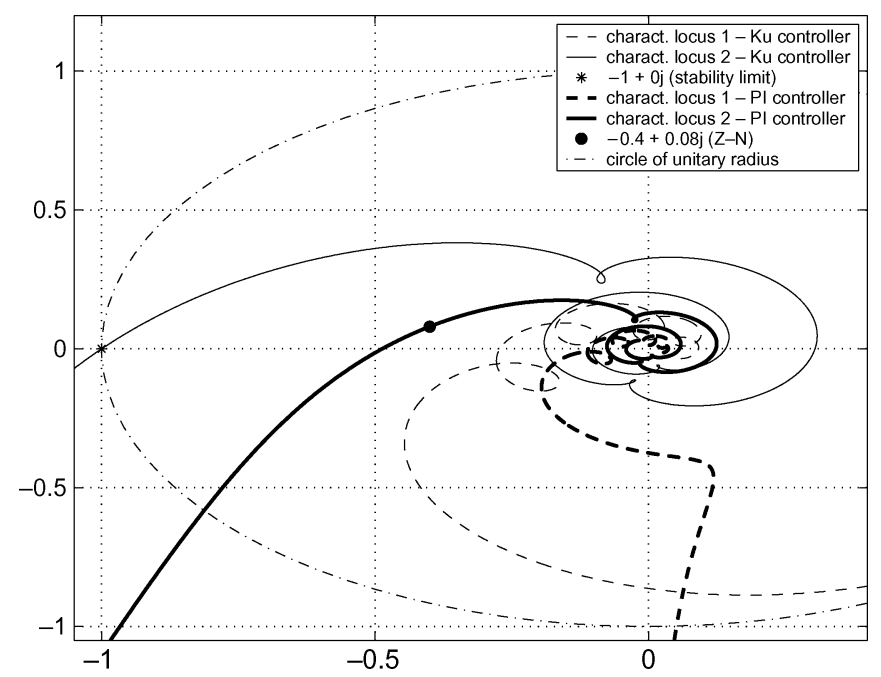

Fig. 12. Characteristic loci of the distillation column with the ultimate gains and with PI controller designed with the proposed method for one characteristic locus.

reached when only one characteristic locus crosses the point $-1+\jmath 0$. If we assume that the other characteristic loci are far enough from -1 , then the insertion of a controller in the loop, however changing the size and shape of them, should not make them involve -1 . In this case, we may concern ourselves only with the "critical" characteristic locus, i.e., the one that crosses -1 , and move only this one to a specified location in the complex plane [13].

We apply this idea to the case study to find a controller that moves the ultimate point of the characteristic locus that crosses the point $-1+0 \jmath$ to $-0.40+0.08 \jmath$, which is the equivalent of Ziegler-Nichols point for SISO ultimate-point method using PI control. Fig. 12 shows the characteristic loci of the system with the ultimate gain and with the PI controller inserted; Fig. 13 shows the step response of the system with the controller obtained using $\hat{G}_{1}\left(\jmath \omega_{u}\right)$; Table VI presents the controller's gains.

The step responses shown in the results present good performance: The overshoot is less than $10 \%$, and the settling time is around $40 \mathrm{~s}$. As shown in Fig. 12, the outermost characteristic
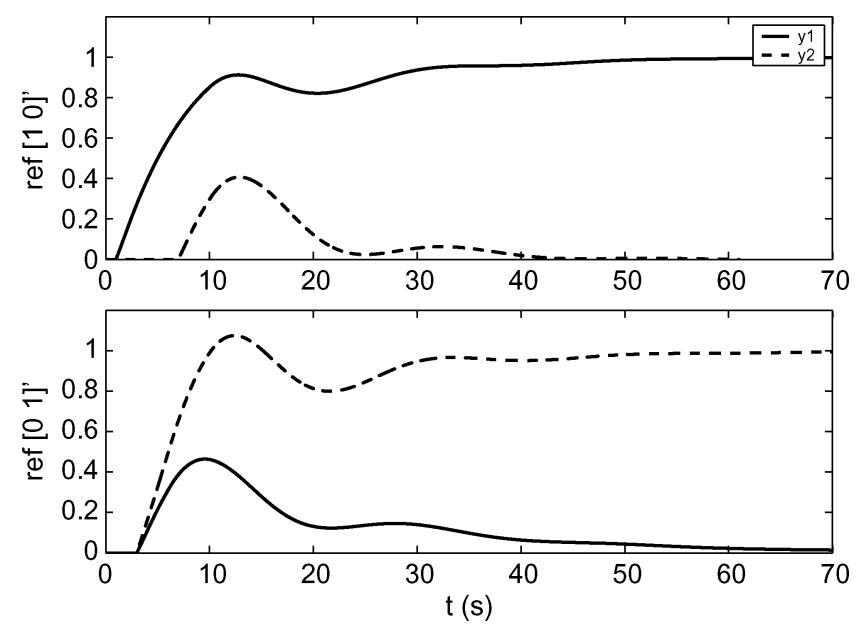

Fig. 13. Step response of the system with PI controller obtained through the multivariable ultimate-point method based on Ziegler-Nichols point.

TABLE VI

PI CONTROLLER's GaINS OF THE SySTEM SHOWn IN Fig. 13

\begin{tabular}{|cccc|}
\hline$k_{p 1}$ & $k_{i 1}$ & $k_{p 2}$ & $k_{i 2}$ \\
\hline 0.1840 & 0.0151 & -0.1214 & -0.0100 \\
\hline
\end{tabular}

locus from the system with PI crosses the circle of unitary radius far from the point $-1+\jmath 0$, and the stability margins can be easily calculated from the Nyquist diagram shown in Fig. 12

$$
\Phi_{M}=38.53^{\circ} \quad G_{M}=2.08 I .
$$

\section{CASE StUdy-PROCESS II}

In this section, we present some results obtained for another process, whose matrix transfer function is presented in (38). This process was also used in [17] and [18] and is considered to have strong coupling

$$
G(s)=\left[\begin{array}{cc}
\frac{0.5}{(0.1 s+1)^{2}(0.2 s+1)^{2}} & \frac{-1}{(0.1 s+1)(0.2 s+1)^{2}} \\
\frac{1}{(0.1 s+1)(0.2 s+1)^{2}} & \frac{2.4}{(0.1 s+1)(0.2 s+1)^{2}(0.5 s+1)}
\end{array}\right]
$$

First of all, we have estimated $\hat{G}\left(\jmath \omega_{u}\right)$ for different ultimate frequencies through DRF tests. Two of them are presented in Table VII.

The controller design for Wood and Berry distillation column, in the previous section, presented better results with the application of the method that tunes a PID which dislocates both characteristic loci to a desired point. This is mainly due to the fact that, regardless of the ultimate point used, similar performances are obtained, i.e., similar stability margins. The choice of the desired point in the complex plane is what determines how large these margins are.

Another important point to study is the influence of the error in the estimation of $\hat{G}\left(\jmath \omega_{u}\right)$. Fig. 14 shows the Nyquist diagrams of the systems controlled by PID 1, obtained with $G\left(\jmath \omega_{u}\right)$ directly from the transfer function (38) and with $\hat{G}_{1}\left(\jmath \omega_{u}\right)$ and $\hat{G}_{2}\left(\jmath \omega_{u}\right)$, which presents a larger estimation error. The design aims to dislocate the ultimate point to $-0.45-0.42 \jmath$. Although the tuning that uses $\hat{G}_{1}\left(\jmath \omega_{u}\right)$ does not reach exactly the desired 
TABLE VII

$\hat{G}\left(\jmath \omega_{u}\right)$ FOR Two DIFFERENT FREQUENCIES OF (38)

\begin{tabular}{|c|cc|c|cc|}
\hline experiment & $\left|u_{2}\right| /\left|u_{1}\right|$ & $\omega_{u}$ & $\hat{G}\left(\omega_{u}\right)$ & $\Delta G$ & $e(\%)$ \\
\hline 1 & 4.0947 & 4.4366 & $\hat{G}_{1}=\left[\begin{array}{ccc}-0.1520-0.1743 \jmath & 0.1502+0.4890 \jmath \\
-0.1531-0.4845 \jmath & -0.5012-0.0631 \jmath\end{array}\right]$ & 0.7 & 0.7 \\
\hline 2 & 0.7544 & 4.4211 & & & \\
\hline 3 & 0.6250 & 4.4927 & $\hat{G}_{2}=\left[\begin{array}{ccc}-0.1736-0.1729 \jmath & 0.1758+0.4491 \jmath \\
-0.1460-0.4981 \jmath & -0.4537-0.0142 \jmath\end{array}\right]$ & 8.4 & 8.3 \\
\hline
\end{tabular}

TABLE VIII

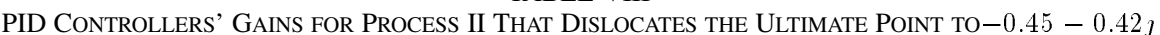

\begin{tabular}{|cc|rrrrrr|rc|}
\hline$G$ & Controller & $k_{p 1}$ & $k_{i 1}$ & $k_{d 1}$ & $k_{p 2}$ & $k_{i 2}$ & $k_{d 2}$ & $G_{M}$ & $\Phi_{M}$ \\
\hline$G\left(\jmath \omega_{u}\right)$ & PID 1 & 5.0803 & 11.4042 & 0.5658 & 0.2066 & 0.8948 & 0.0119 & $2.44 I$ & $24.16^{\circ}$ \\
$G\left(\jmath \omega_{u}\right)$ & PID 2 & 0.1156 & 2.4170 & 0.0014 & 1.7771 & 1.7969 & 0.4394 & $2.66 I$ & $40.47^{\circ}$ \\
\hline$\hat{G}_{1}\left(\jmath \omega_{u}\right)$ & PID 1 & 5.1997 & 11.5929 & 0.5830 & 0.2097 & 0.9036 & 0.0122 & $2.41 I$ & $24.77^{\circ}$ \\
$\hat{G}_{1}\left(\jmath \omega_{u}\right)$ & PID 2 & 0.1244 & 2.4665 & 0.0016 & 1.7836 & 1.7843 & 0.4457 & $2.60 I$ & $39.85^{\circ}$ \\
\hline$\hat{G}_{2}\left(\jmath \omega_{u}\right)$ & PID 1 & 4.9476 & 10.4319 & 0.5866 & 0.2372 & 1.0516 & 0.0134 & $2.50 I$ & $16.40^{\circ}$ \\
$\hat{G}_{2}\left(\jmath \omega_{u}\right)$ & PID 2 & 0.1033 & 2.4329 & 0.0011 & 1.8223 & 1.6191 & 0.5127 & $2.84 I$ & $42.36^{\circ}$ \\
\hline
\end{tabular}

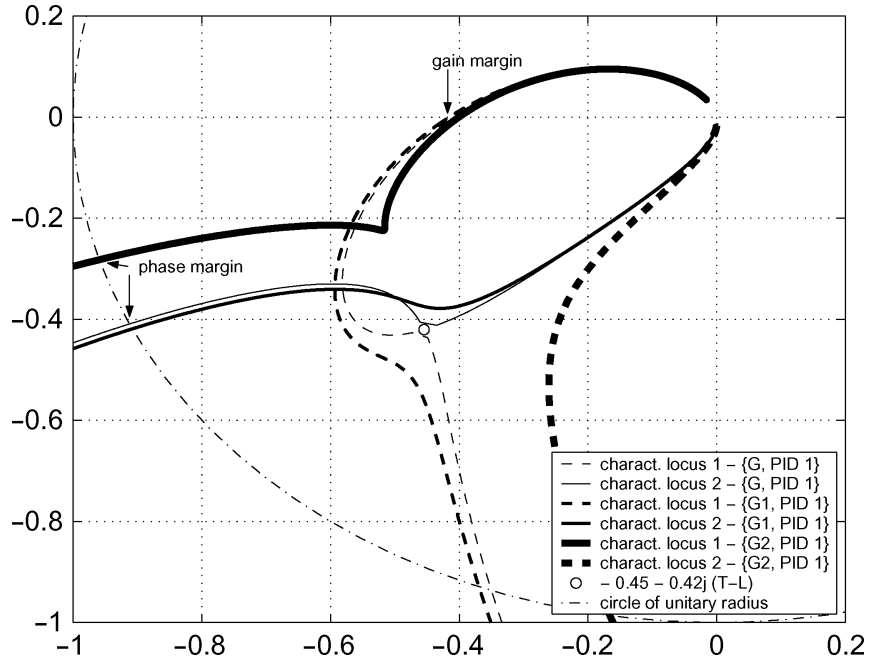

Fig. 14. Characteristic loci of Process II with PID 1, when using the real $G\left(\jmath \omega_{u}\right)$ and the estimations $\hat{G}_{1}\left(\jmath \omega_{u}\right)$ and $\hat{G}_{2}\left(\jmath \omega_{u}\right)$.

point, the resulting stability margins are indistinguishable from the ones obtained with the real $G\left(\jmath \omega_{u}\right)$. The tuning obtained for $\hat{G}_{2}\left(\jmath \omega_{u}\right)$ places the ultimate point even farther from the desired location. In this case, the gain margin has been unchanged, but the phase margin has been decreased significantly for PID 1.

Besides, Fig. 15 shows the Nyquist diagrams for the same design, but in this case, we compare the design results when PID 2 is applied. It can be seen that the estimation error has a large influence in the tuning method, which is unable to reach the desired point in the complex plane, but in the other hand, the stability margins have not been changed significantly. Table VIII presents the controller gains, obtained with $G\left(\jmath \omega_{u}\right), \hat{G}_{1}\left(\jmath \omega_{u}\right)$, and $\hat{G}_{2}\left(\jmath \omega_{u}\right)$, as well as the stability margins obtained with each controller. It can be seen that PID 2 seems to be the best controller whatever estimation of $G\left(\jmath \omega_{u}\right)$ is used for this process. Thus, if we choose PID 2, there is no significant influence of the estimation error in the performance of the system. This is

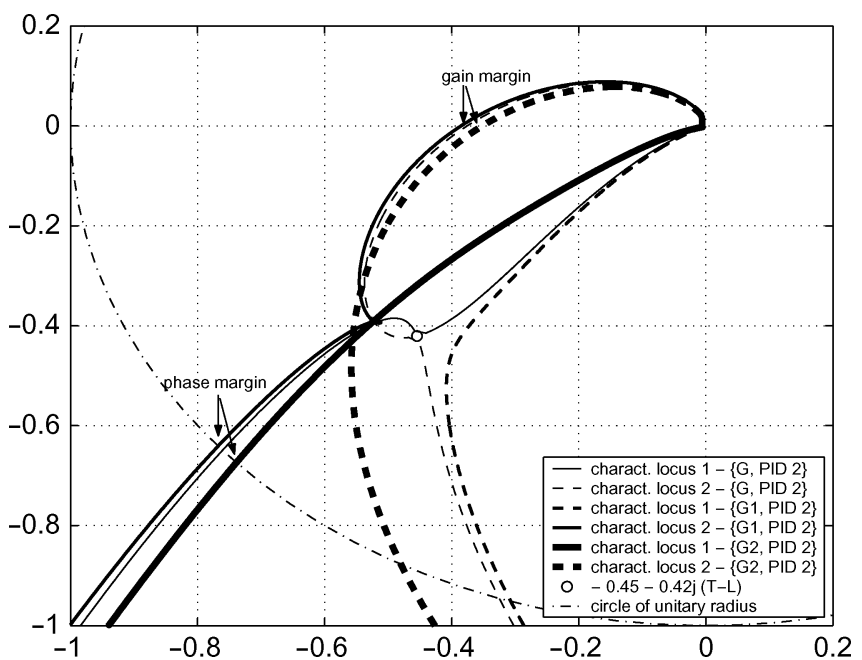

Fig. 15. Characteristic loci of Process II with PID 2, when using the real $G\left(\jmath \omega_{u}\right)$ and the estimations $\hat{G}_{1}\left(\jmath \omega_{u}\right)$ and $\hat{G}_{2}\left(\jmath \omega_{u}\right)$.

also shown in Figs. 16 and 17, which present the system response when the controller PID 2 is applied and the estimations $\hat{G}_{1}\left(\jmath \omega_{u}\right)$ and $\hat{G}_{2}\left(\jmath \omega_{u}\right)$, respectively.

\section{CONCLUSION}

An extension of the ultimate-point method for multivariable systems has been proposed, which consists in dislocating the ultimate point to a given location on the complex plane. Different locations on the complex plane can be chosen. In the SISO case, this chosen location is related to the stability margins of the closed-loop system, provided that some assumptions are satisfied by the frequency response of the process. The same assumptions, when made for the characteristic loci of a MIMO process, will imply the same properties of stability margins. The method proposed inherits the virtues that has made the SISO ultimate-point method very successful but also its shortcomings. Although we have applied this method successfully to a number of relevant examples, it is not clear at this point for 

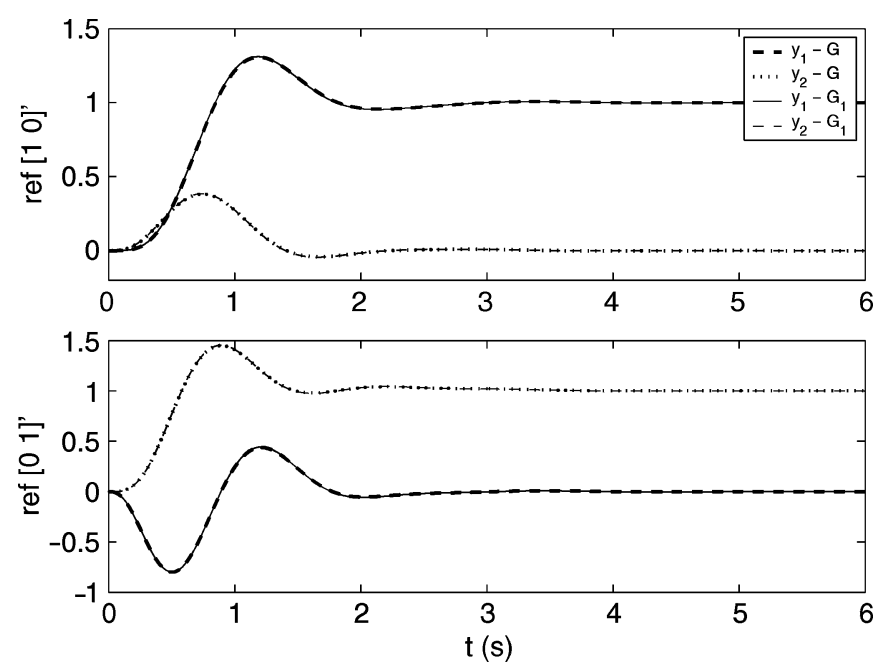

Fig. 16. Step response of Process II with PID 2 obtained with the real $G\left(\jmath \omega_{u}\right)$ and $\hat{G}_{1}\left(\jmath \omega_{u}\right)$.
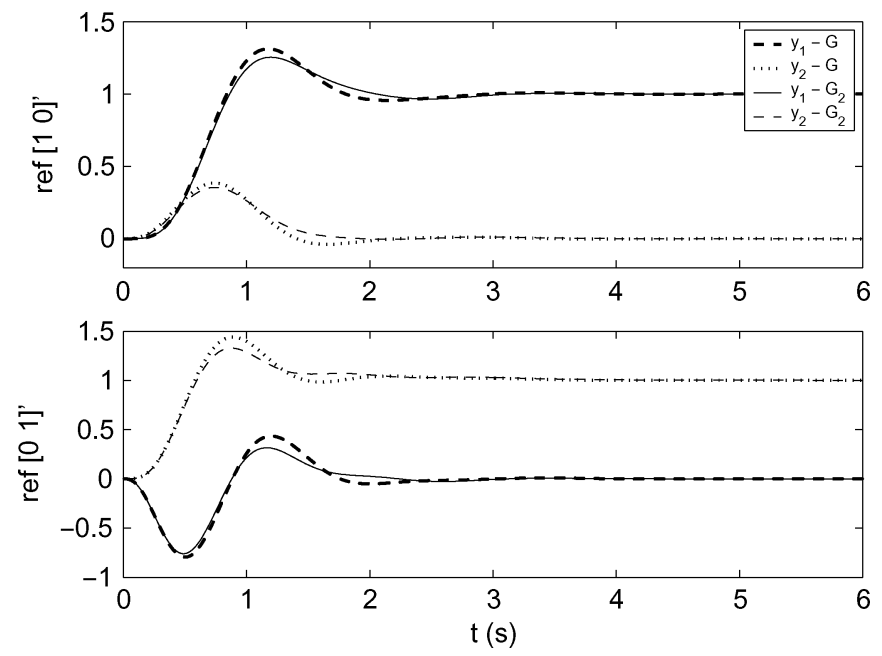

Fig. 17. Step response of Process II with PID 2 obtained with the real $G\left(\jmath \omega_{u}\right)$ and $\hat{G}_{2}\left(j \omega_{u}\right)$.

which classes of MIMO systems these assumptions are satisfied and how generic these properties are. This is an important area of future research.

On the other hand, previously reported tuning methods for MIMO systems based on the MIMO ultimate quantities are strongly dependent of the particular ultimate point identified. These designs can fail if a "bad" ultimate point is identified, and there are no guidelines to prevent this situation. Our method does not suffer from this drawback, as the use of different ultimate points for the design leads to quite similar performance, as can be seen by the stability margins presented in Tables $\mathrm{V}$ and VIII.

\section{REFERENCES}

[1] K. Åström and T. Hagglund, PID Controllers: Theory, Design and Tuning, 2nd ed. Research Triangle Park, NC: Instrum. Soc. Amer., 1995.
[2] C.-C. Yu, Autotuning of PID Controllers: Relay Feedback Approach. London, U.K.: Springer-Verlag, 1999.

[3] J. Ziegler and N. Nichols, "Optimum settings for automatic controllers," Trans. ASME, vol. 64, pp. 759-768, 1942.

[4] D. E. Seborg, T. F. Edgar, and D. A. Mellichamp, Process Dynamics and Control, 2nd ed. New York: Wiley, 2004.

[5] S. Skogestad and I. Postlethwaite, Multivariable Feedback Control: Analysis and Design, 2nd ed. New York: Wiley, 2005.

[6] A. Loh, C. Quek, and V. Vasnani, "An approach to multivariable control system design using relay auto-tuning," in Proc. Singapore Int. Conf. Intell. Control Instrum., Singapore, 1992, vol. 1, pp. 162-164.

[7] S. Menani and H. N. Koivo, "Automatic tuning of multivariable controllers with adaptive relay feedback," in Proc. 35th Conf. Decision Control, Kobe, Japan, Dec. 1996, vol. 4, pp. 4695-4700.

[8] J. Maciejowski, Multivariable Feedback Design. Wokingham, U.K.: Addison-Wesley, 1989.

[9] H. N. Koivo, "A multivariable self-tuning controller," Automatica, vol. 16, no. 4, pp. 351-366, Jul. 1980.

[10] Y. Halevi, Z. J. Palmor, and T. Efrati, "Automatic tuning of decentralized PID controllers for MIMO processes," J. Process Control, vol. 7, no. 2, pp. 119-128, Apr. 1997.

[11] A. P. Loh and V. Vasnani, "Describing function matrix for multivariable systems and its use in multiloop PI design," J. Process Control, vol. 4, no. 3, pp. 115-120, 1994.

[12] D. Semino, L. Mazzanti, and C. Scali, "Design of decentralized controllers by a relay technique: Extensions of tuning rules," in Proc. UKACC Int. Conf. Control, 1996, vol. 427, pp. 1190-1195.

[13] L. Campestrini and A. S. Bazanella, "Tuning of multivariable PID controllers through the ultimate point method," in Proc. 45th IEEE CDC, San Diego, CA, 2006, vol. 1, pp. 1834-1839.

[14] L. Campestrini, P. R. Barros, and A. S. Bazanella, "Auto-tuning of PID controllers for MIMO processes by relay feedback," in Proc. ADCHEM, Gramado, RS, Brazil, 2006, vol. 1, pp. 451-456.

[15] W. Luyben, B. Tyréus, and M. Luyben, Plantwide Process Control, 2nd ed. New York: McGraw-Hill, 1998.

[16] W. K. Ho, T. H. Lee, W. Xu, J. R. Zhou, and E. B. Tay, "The direct Nyquist array design of PID controllers," IEEE Trans. Ind. Electron., vol. 47, no. 1, pp. 175-185, Feb. 2000.

[17] Z. J. Palmor, Y. Halevi, and N. Krasney, "Automatic tuning of decentralized PID controllers for TITO processes," Automatica, vol. 31, no. 7, pp. 1001-1010, Jul. 1995.

[18] Q.-G. Wang, B. Zou, T.-H. Lee, and Q. Bi, "Auto-tuning of multivariable PID controllers from decentralized relay feedback," Automatica, vol. 33, no. 3, pp. 319-330, Mar. 1997.

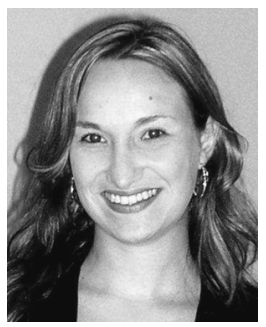

Lucíola Campestrini was born in Erechim, Brazil, in 1981. She received the Engineer's degree in control and automation engineering from Pontifícia Universidade Católica do Rio Grande do Sul, Porto Alegre, Brazil, and the M.Sc. degree in electrical engineering with research subject on decentralized multivariable control from the Universidade Federal do Rio Grande do Sul, Porto Alegre, in 2005, where she is currently working toward the Ph.D. degree with main research subjects on data-based control systems and experiment design in the Electrical

Engineering Department.

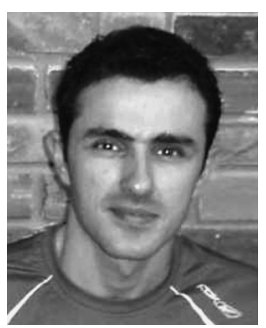

Luiz Carlos Stevanatto Filho was born in 1975. He received the B.S. degree in electrical engineering from Escola de Engenharia Mauá, São Caetano do Sul, Brazil, in 1998 and the M.Sc. degree in electrical engineering with research subject on multivariable system identification through decentralized relays from the Universidade Federal do Rio Grande do Sul, Porto Alegre, Brazil, in 2008.

Since 2003, he has been with the Universidade de Caxias do Sul, Caxias do Sul, Brazil, where he is currently with the Engineering Laboratory in the Mechanical Engineering Department. His research interests include low-cost system identification and automatic controller tuning. 


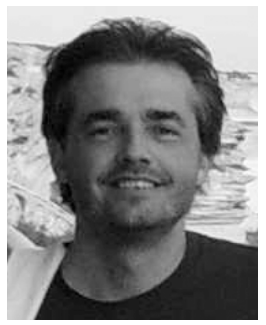

Alexandre Sanfelice Bazanella was born in Porto Alegre, Brazil, in 1969. He received the Ph.D. degree in electrical engineering from the Universidade Federal de Santa Catarina, Santa Catarina, Brazil, in 1997.

$\mathrm{He}$ is currently an Associate Professor with the Electrical Engineering Department, Universidade Federal do Rio Grande do Sul, Porto Alegre, Brazil. He was a Visiting Professor at the Universidade Federal da Paraiba, Paraiba, Brazil, and at the Université Catholique de Louvain, Louvain-la-Neuve, Belgium, where he spent a sabbatical year in 2006. His current main research interest are in system identification and data-based control design. He has also authored a number of papers in nonlinear systems theory, particularly its application to electric power systems and electrical machines, and an undergraduate textbook in control systems theory.

Dr. Bazanella has been serving as Associate Editor for the IEEE TRANSACTIONS ON CONTROL SYSTEMS TECHNOLOGY. 